\title{
UTILIZAÇÃO DO CANTO GREGORIANO NA SAÚDE: UMA REVISÃO BIBLIOGRÁFICA NARRATIVA
}

Ana Paula Almeida ${ }^{1}$, Maria Julia Paes da Silva²

\begin{abstract}
RESUMO: O objetivo deste estudo foi de revisar a literatura científica com vistas à identificação das publicações sobre o canto Gregoriano na área da saúde. Trata-se de um estudo de revisão bibliográfica narrativa com abordagem quantitativa. Os dados foram coletados no período de janeiro a abril de 2011, sem limite de tempo e de idioma. Totalizou-se 149 artigos sendo que destes, foram analisados 128 na íntegra, pois 21 artigos não foram localizados. Desta análise, nenhum artigo atendeu ao critério de inclusão, ou seja, não havia citação do canto Gregoriano como música para intervenção em cuidado à saúde. Considerando-se que o canto Gregoriano mostra-se como um tema inovador para o cuidado à saúde, é preciso fazer uma revisão dos descritores e realizar pesquisas para refinar suas potencialidades.
\end{abstract}

PALAVRAS-CHAVE: Música; Hospitalização; Terapias complementares; Saúde.

\section{USE OF THE GREGORIAN CHANT IN HEALTH: A NARRATIVE BIBLIOGRAPHICAL REVIEW}

\begin{abstract}
The objective of this study was to review the scientific literature with a view to identifying the publications about the Gregorian chant in the area of health. It is a bibliographical narrative review study with a quantitative approach. The data was collected in the period January - April 2011, without time or language limits. In total there were 149 articles of which 128 were analyzed in full, as 21 articles were not located. In this analysis, no article met the inclusion criteria, that is, the Gregorian chant was not cited as music for intervention in healthcare. Considering that the Gregorian chant is an innovative issue in healthcare, it is necessary to undertake a review of the descriptors and carry out research to refine its potential.

KEYWORDS: Music; Hospitalization; Complementary therapies; Health.

\section{UTILIZACIÓN DEL CANTO GREGORIANO EN LA SALUD: UNA REVISIÓN BIBLIOGRÁFICA NARRATIVA}

RESUMEN: El objetivo de este estudio fue revisar la literatura científica a fin de identificar las publicaciones acerca del Canto Gregoriano en el área de salud. El estudio es una revisión bibliográfica narrativa con abordaje cuantitativo. Los datos fueron recogidos en el periodo de enero a abril de 2011, sin límite de tiempo y de idioma. El resultado fue un total de 149 artículos siendo analizados en la íntegra, pues 21 artículos no fueron ubicados. De este análisis, ningún artículo obedeció al criterio de inclusión, o sea, no había citación del Canto Gregoriano como música para intervención en cuidado a la salud. Considerando que el Canto Gregoriano es un tema innovador para el cuidado a la salud, son necesarias una revisión de los descriptores así como investigaciones para perfeccionar sus potencialidades.

PALABRAS CLAVES: Música; Hospitalización; Terapías complementares; Salud.

\footnotetext{
${ }^{1}$ Enfermeira do Serviço de Educação Permanente do Instituto da Criança - Hospital das Clínicas da Faculdade de Medicina da Universidade de São Paulo. Mestre em Ciências.

${ }^{2}$ Enfermeira. Professora do Departamento de Enfermagem Médico-Cirúrgica da Escola de Enfermagem da Universidade de São Paulo. Líder do Grupo de Estudo Práticas Alternativas ou Complementares em Saúde.
}

Autor correspondente:

Recebido: 08/09/2011

Ana Paula Almeida Aprovado: 16/04/2012

Universidade de São Paulo

Rua Marquês de Paranaguá, 51- 01303-050 - São Paulo-SP-Brasil

E-mail: anaflumian@gmail.com

Cogitare Enferm. 2012 Jul/Set; 17(3):556-61 


\section{INTRODUÇÃO}

O som é uma série de vibrações provocadas em corpos elásticos e transmitidas aos meios elásticos vizinhos. Estas vibrações, quando regulares, comprovadas por meios gráficos são chamadas de sons. Ao contrário, constituem os ruídos. Assim, conclui-se que, sendo exigida uma medida para a determinação do som, fica fácil de entender que a música pode ser um problema matemático, além de físico. Sabe-se que o conjunto de sons dá formação à música que existe em todos os tempos como expansão da humanidade, assim como as outras artes e modalidades da linguagem. Desde o homem primitivo descobrindo e usando sua voz, primeiro instrumento de sopro ao alcance do Homem até os dias atuais, com a mais requintada tecnologia sonora, a Humanidade utiliza a música para expressar suas emoções, desejos e ideias ${ }^{(1)}$.

A música afeta as necessidades físicas, emocionais, cognitivas e sociais do indivíduo em todas as faixas etárias. Tem efeitos benéficos no alívio da dor ${ }^{(2,3)}$, diminuindo o estresse e a ansiedade pré-operatória em crianças $^{(4)}$, age de forma benéfica em crianças no pósoperatório de cirurgia cardíaca, verificado por meio dos sinais vitais - frequência cardíaca e respiratória, bem como na redução da dor verificada com a utilização da escala facial de dor ${ }^{(2)}$.

A convivência com a música ocorreu desde a infância, com o sonho dos meus pais em ver suas filhas tocando piano. O piano permitiu o contato com a música sacra. Informalmente, diz-se que o canto Gregoriano nos faz acalmar.

O canto Gregoriano é a mais antiga música ocidental em uso nos nossos dias, datada no fim do século VI. As qualidades características do coral gregoriano são a inesgotável riqueza melódica, o ritmo puramente prosódico, subordinado ao texto, dispensa a separação dos compassos pelo risco. É rigorosamente homofônica, mesmo se cantada por um coro numeroso. Ouve-se sempre em uníssono ${ }^{(5)}$.

O canto Gregoriano tem a capacidade de reduzir a ansiedade, pois respeita a frequência respiratória ${ }^{(6)}$. Os intervalos reduzidos que o canto Gregoriano mantém, de acordo com a frequência respiratória e a acentuação das palavras em latim, fixa a atenção do ouvinte durante sua execução( ${ }^{(7)}$. Neste gênero musical não há momentos de tensão ou clímax que se faça esperar, o que poderia nos deixar cansados. Ao contrário, sua linha musical é sustentada. $O$ fato de não termos familiaridade com o latim torna difícil saber qual seria nossa reação ao saber sobre o tema e desenvolvimento da canção. Talvez a intenção seja de nos agradar somente com a presença serena e elevada desta música ${ }^{(6)}$. De acordo com o descrito, pode-se inferir um efeito benéfico, de forma complementar no tratamento de transtorno da ansiedade com a audição do canto Gregoriano.

Na história da Enfermagem, a música começou a ser utilizada com a finalidade terapêutica por Florence Nightingale em 1859. Florence Nightingale já mencionava que a utilização da música era um cuidado à saúde e que

os instrumentos de sopro, inclusive a voz humana e os instrumentos de corda capazes de produzir sons contínuos, em geral trazem efeitos benéficos e isso independentemente da associação com o sentido dessas $\operatorname{melodias}^{(8: 66)}$.

Isa Maud Ilsen, musicista e enfermeira, foi a responsável pela criação da Associação Nacional de Música nos Hospitais, pioneira no ensino de musicoterapia na Universidade de Columbia e Harryet Ayer Seymour, também enfermeira, depois de observar soldados feridos das I e II Guerras Mundiais, dedicou-se a terapêutica musical por meio de método próprio, o "Método Seymour". Preocupada com o tema, abriu uma escola para formação de musicistas para tocar a beira do leito de pessoas hospitalizadas e fez vários concertos para milhares de doentes nos Estados Unidos ${ }^{(1,9)}$.

O COFEn, por meio da Resolução 197, estabelece e reconhece as Terapias Alternativas como especialidade e/ou qualificação profissional de Enfermagem. No Parecer Normativo 004/95, aprovado na 239 ${ }^{\mathrm{a}}$ Reunião Ordinária realizada em 18.07.95, dispõe que as terapias alternativas (Acupuntura, Iridologia, Fitoterapia, Reflexologia, Quiropraxia, Massoterapia, dentre outras), são práticas oriundas, em sua maioria, de culturas orientais exercidas ou executadas por práticos treinados sem sistematização e são conhecimentos repassados de geração em geração, não estando vinculados a qualquer categoria profissional. Porém, para receber esta titulação, o profissional de Enfermagem deverá ter concluído e sido aprovado em curso reconhecido por instituição de ensino ou entidade congênere, com uma carga horária mínima de 360 horas ${ }^{(10)}$.

Por ainda termos pouco contato com esse tipo de música no cuidado complementar dos pacientes, pareceu-nos necessária uma revisão sobre o tema, com vistas a estimular ou não mais pesquisas sobre o uso dessa música pela Enfermagem. 


\section{MÉTODO}

Trata-se de um estudo de revisão narrativa da literatura que buscou obter uma visão abrangente das publicações sobre a utilização do canto Gregoriano no cuidado à saúde; para este fim, o estudo valeu-se da revisão narrativa tradicional. Esta revisão sintetiza e resume, em termos narrativos, um corpo da literatura de pesquisa científica. Optou-se por este método de investigação científica pelos critérios de confiabilidade tanto para a literatura nacional quanto internacional ${ }^{(11)}$.

Nesta revisão buscou-se incluir todos os artigos publicados sobre a utilização do canto Gregoriano no cuidado à saúde em todos os periódicos indexados nas bases de dados nacionais e internacionais, referenciadas e reconhecidas na área de pesquisa em saúde, como segue: LILACS (Literatura latino-Americana e do Caribe em Ciências da Saúde), EMBASE (Excerpta Medica Database) e PubMed (US National Library of Medicine).

$\mathrm{O}$ canto Gregoriano não é um Descritor de Ciências em Saúde (DeCS), o que dificultou a busca por trabalhos que o citem. A criação de termos para inclusão no DeCS pode ser proposta para atender as áreas temáticas ainda não contempladas nas categorias já existentes e que possam ser utilizados em qualquer país. Os termos podem ser substantivos simples ou compostos com frequência comprovada por meio de, no mínimo, 10 citações na literatura nacional e internacional e estar claramente inserido no contexto do domínio que pretende descrever. Quando um termo for muito específico, será válido somente se o critério frequência justificar sua criação ${ }^{(12)}$. Assim, pelo critério frequência, canto Gregoriano pode ser um termo considerado específico.

Para iniciar esta revisão, foram utilizados os descritores Music, Hospitalization, Complementary Therapies e Health. Não foi utilizado limite temporal nem de idioma; os indexadores foram agregados pelos dispositivos de buscas $A N D$ para separar um termo do outro. Excluíram-se os artigos que, apesar de mencionarem o canto Gregoriano, este não era o objeto de estudo da pesquisa.

Alguns artigos estavam disponibilizados na íntegra pela Internet e outros foram localizados na Biblioteca Virtual em Saúde (BVS), Biblioteca da Escola de Enfermagem da USP e da Faculdade de Medicina da Universidade de São Paulo. Alguns artigos citados ${ }^{(12)}$ não foram localizados; buscou-se por endereço para correspondência com os autores dos trabalhos não localizados, mas não houve sucesso. Os dados foram coletados no período de janeiro a abril de 2011.

Para organizar a análise dos artigos foi elaborada uma ficha composta pelos seguintes campos: título do artigo, nome dos autores, ano de publicação, disponibilidade de acesso (resumo ou texto completo), base de dados (LILACS, EMBASE, PubMed), categoria profissional dos autores (Enfermagem, Medicina, Psicologia, Outros), país de origem do estudo (Brasil, Reino Unido, Cuba, Argentina, Estados Unidos da América, Alemanha, Outros), tipo de publicação (artigos originais, revisão, reflexão, atualização, resenha e relato de experiência), objetivo da pesquisa (descrever/ avaliar, testar intervenção, validar instrumento, revisar literatura, outro), tipo de pesquisa (qualitativa ou quantitativa), população estudada (geral ou específica), número da amostra, conclusão, observações necessárias e descritores.

\section{RESULTADOS}

Após uma busca bibliográfica minuciosa com análise de título, resumo e material na íntegra, o resultado revelou-se como descrito na tabela 1 .

Tabela 1 - Artigos levantados, analisados e selecionados sobre canto Gregoriano de acordo com as bases de dados. São Paulo, 2011

\begin{tabular}{lcccccc}
\hline Bases de dados & \multicolumn{2}{c}{$\begin{array}{c}\text { Estudos levantados } \\
\text { \% }\end{array}$} & \multicolumn{2}{c}{ Estudos analisados } & \multicolumn{2}{c}{ Estudos selecionados } \\
& $\mathbf{N}$ & $\mathbf{N}$ & $\mathbf{\%}$ & $\mathbf{N}$ & $\mathbf{\%}$ \\
\hline LILACS & 69 & 43,3 & 69 & 53,9 & 0 & 0 \\
EMBASE & 21 & 14,1 & 15 & 11,8 & 0 & 0 \\
PubMeb & 59 & 39,6 & 44 & 34,3 & 0 & 0 \\
Total & 149 & $100^{*}$ & 128 & $100^{*}$ & 0 & 0 \\
\hline
\end{tabular}

*valores ajustados para $100 \%$ 
Após a busca criteriosa pelos artigos nos bancos de dados citados anteriormente, totalizou-se 149 artigos que responderam aos DeCS. Destes, foram analisados 128 artigos na íntegra, pois 21 artigos não foram localizados (6 citados na EMBASE e 21 no PubMed). Desta análise, nenhum artigo atendeu ao critério de inclusão, ou seja, não se referia ao cuidado na saúde com a utilização do canto Gregoriano.

\section{DISCUSSÃO}

Esperava-se que, com os DeCS selecionados, fosse possível encontrar artigos que tivessem o canto Gregoriano como uma terapia musical a ser explorada. $\mathrm{O}$ canto Gregoriano é uma música que parece não precisar de referências para ser enaltecida como um tipo de canto que faz o Homem ter momentos de grande paz interior enquanto o ouve. Infere-se que, talvez assim seja por ser um estilo de música antiga e por fazer parte da prática diária de sacerdotes com a intenção de se encontrar com Deus.

Em pesquisa bibliográfica nacional com objetivo de analisar estudos que abordam a utilização da música na enfermagem verificou que em 7 de 12 estudos, o estilo de música aplicado nas pesquisas foi a música clássica $^{(13)}$. Desta forma, há de se considerar outros estilos musicais pouco explorados no cuidado a saúde e, entre eles, está a música sacra.

Em estudos que não foram encontrados com esta busca bibliográfica, o canto Gregoriano é utilizado como recurso diagnóstico de problemas neurológicos. Pesquisadores brasileiros utilizaram o canto Gregoriano para mapeamento eletroencefalográfico de dois pacientes afásicos do sexo masculino. Importante ressaltar que, neste estudo, foi encontrada grande proporção de frequência beta - sono profundo - e potência no hemisfério não lesado. Também foi encontrado aumento na frequência de atividade alfa - relaxamento - no hemisfério cerebral não lesado restrito à região do lobo temporal. Esta pesquisa foi encontrada no PubMed, porém, não aparece com a busca realizada utilizando os descritores anteriormente citados ${ }^{(14)}$.

Outra autora brasileira utilizou o canto Gregoriano para realizar uma análise quantitativa da atividade elétrica em pacientes epiléticos; 30 indivíduos adultos (20 indivíduos epilépticos e 10 não epilépticos) foram submetidos a estimulações cognitivas durante a captação dos ritmos elétricos cerebrais. Para o estudo, o grupo foi constituído por 10 homens e 10 mulheres portadores de epilepsia temporal parcial. No grupo controle havia 5 homens e 5 mulheres sem história de doença neurológica prévia ou atual. Contudo, os indivíduos do grupo epiléptico e do grupo controle foram controles de si mesmos, uma vez que foram avaliados os traçados do eletrencefalograma antes e durante a estimulação. As estimulações foram compostas por testes dicótico verbal, cálculos matemáticos, e audição de canto Gregoriano. Parece interessante ter encontrado no grupo controle masculino uma diminuição da frequência beta - sono profundo - em todas as regiões analisadas e, no grupo controle feminino um aumento da mesma frequência beta. No grupo epiléptico masculino esquerdo, durante a audição do canto Gregoriano, houve uma tendência à diminuição da frequência alfa, diferente do que ocorreu com o grupo controle masculino. Este trabalho é resultado de uma tese de Doutorado de uma enfermeira brasileira e não foi encontrada uma publicação em forma de artigo. Este estudo utilizou os descritores Eletroencefalografia e Epilepsia motora parcial ${ }^{(15)}$. De acordo com o resultado deste estudo, infere-se que a audição de canto Gregoriano proporcionou maior relaxamento nas mulheres.

Um terceiro estudo com 70 estudantes investigou se ouvir música interfere no nível de testosterona em mulheres. Foram realizados estímulos por trinta minutos em grupos divididos para ouvir a música favorita, o silêncio, o canto Gregoriano, a música popular, jazz e Mozart. Esta pesquisa revelou que o canto Gregoriano diminuiu o nível da testosterona, em relação ao silêncio, verificada por meio dos níveis deste hormônio na saliva. É sabido que a testosterona pode influenciar o comportamento agressivo e vice-versa. Este hormônio está relacionado com a libido, nível de atividade, busca de sensações e desempenha importante papel nos comportamentos de agressividade humana. Desta forma, acredita-se que, com a diminuição da testosterona na mulher provocada pela audição de canto Gregoriano, seu uso influencie positivamente no relaxamento. $\mathrm{O}$ estudo utilizou as palavras-chave music e testoterone ${ }^{(16)}$.Verificou-se ainda que, o canto Gregoriano foi objeto de estudo em pesquisa de mestrado, cujo objetivo foi verificar se o mesmo altera o estado de ansiedade das mães com filhos internados. Foi realizada uma pesquisa descritiva, exploratória, correlacional com análise quantitativa, quase experimental. Setenta e uma mães foram convidadas para participar do estudo. Realizaram-se duas audições musicais com intervalo de 48 horas entre a primeira e a segunda. Utilizou-se o Inventário de Diagnóstico de Ansiedade Traço-estado (IDATE) como instrumento de medida da ansiedade das mães. Na primeira audição musical 
verificou-se que 25(89,2\%) mulheres tiveram seu estado de ansiedade diminuídos e na segunda audição musical, também 25(89,2\%) continuaram a ter seu estado de ansiedade diminuídos. Vinte e oito mães concluíram a pesquisa. Sem generalizações devido o número de mães estudadas, pôde-se concluir que a utilização do canto Gregoriano diminuiu o estado de ansiedade das mães de crianças hospitalizadas em um hospital pediátrico de atenção quaternária acomodados em quartos individuais. Os descritores deste estudo foram: Música, Ansiedade, Mães, Terapias Complementares, Terapias Alternativas ${ }^{(17)}$.

\section{CONSIDERAÇÕES FINAIS}

Com esse resultado, para ter-se uma busca bibliográfica com resultado positivo, no sentido de recuperar dados de pesquisa que inclua o canto Gregoriano como objeto primário ou secundário de estudo, parece ser necessário fazer uma revisão dos descritores utilizados nas pesquisas e realizar novas buscas.

Como o canto Gregoriano mostra-se como um tema inovador para o cuidado à saúde, é preciso apurar sua utilização em outras pesquisas para refinar suas potencialidades.

É sabido que a música, como terapia complementar, é uma grande aliada na assistência de Enfermagem, além de se revelar um modo simples de autocuidado, ser de baixo custo e disponível à população. $\mathrm{O}$ que $\mathrm{o}$ canto Gregoriano pode fazer no sentido de ampliar o potencial desse universo terapêutico, ainda é uma incógnita para a Enfermagem e para as áreas de saúde, de uma maneira geral.

\section{REFERÊNCIAS}

1. Ribas JC. Música e Medicina. São Paulo: Edigraf; 1957.

2. Hatem TP, Lira PI, Mattos SS. The therapeutic effects of music in children following cardiac surgery. J. pediatr. [Internet]. 2006;82. [acesso 24 abr 2011]. Disponível: http://www.scielo.br/pdf/jped/v82n3/v82n3a06.pdf

3. Zárate PD, Diaz VT. Aplicaciones de la musicoterapia em la medicina. Rev. Méd. Chile. [Internet]. 2001;129(2). [acesso em 24 abr 2011]. Disponível: http://www.scielo.cl/scielo.php?pid=S0034$98872001000200015 \&$ script=sci_arttext\&tlng=en

4. Kain ZN, Caldwell-Andrews AA, Krivutza DM, Weinberg ME, Gaal D, Wang SM, et al. Interactive Music therapy as a treatment for preoperative anxiety in children: a randomized controlled trial. Anesth. analg. [Internet].
2004;98. [acesso em 10 mar 2011]. Diponível: http://www. anesthesia-analgesia.org/content/98/5/1260.short

5. Carpeaux OM. O livro de ouro da História da Música: da idade média ao século XX. $3^{\mathrm{a}}$ ed. Rio de Janeiro: Ediouro; 2001.

6. Campbell D. O efeito Mozart: explorando o poder da música para curar o corpo, fortalecer a mente e liberar a criatividade. Rio de Janeiro: Rocco; 2001.

7. Le Mée KW. Canto: as origens, a forma, a prática e o poder curativo do canto gregoriano. Rio de Janeiro: Agir; 1996.

8. Nightingale F. Notas em enfermagem: o que é e o que não é. São Paulo: Cortez; 1989. Ruídos.

9. Leão ER, Silva MJP. Música e dor crônica musculoesquelética: o potencial evocativo de imagens mentais. Rev. Latino-Am. Enferm. [Internet]. 2004;2(2). [acesso em 12 mar 2011]. Disponível: http://www.scielo. br/pdf/rlae/v12n2/v12n2a13.pdf

10. Conselho Federal de Enfermagem (BR). Resolução COFEN n. 197. Estabelece e reconhece as terapias alternativas como especialidade e/ou qualificação do profissional de Enfermagem. In: Conselho Regional de Enfermagem. Documentos básicos de enfermagem. São Paulo; 1997.

11. Polit DF, Beck CT. Fundamentos de pesquisa em enfermagem: avaliação de evidências para a prática de enfermagem. Porto Alegre: Artmed; 2011.

12. Bireme. Guia para atualização do DeCS. $1^{\mathrm{a}}$ versão. [Internet]. 2007. [acesso em 28 jul 2011]. Disponível: http://bvsmodelo.bvsalud.org/download/decs/DeCSGuiaAtualizacao.pdf

13. Gonçalves DFC, Nogueira ATO, Puggina ACG. Uso da música na assistência de enfermagem no Brasil: um revisão bibliográfica. Cogitare enferm. [Internet]. 2008;13(4). [acesso em 29 jul 2011]. Disponível: http:// ojs.c3sl.ufpr.br/ojs-2.2.4/index.php/cogitare/article/ view/13121/8881

14. Muszkat M, Correia CMF, Noffs MHS, Vincenzo NS, Campos CJR. Especialização funcional hemisférica da afasia motora. Arq. neuropsiquiatr. [Internet]. 1995;53(1). [acesso em 23 fev 2011]. Disponível: http:// www.scielo.br/pdf/anp/v53n1/14.pdf

15. Frayman L. Análise quantitativa da atividade elétrica cerebral durante estimulação cognitiva de pacientes 
epiléticos [tese]. São Paulo (SP): Universidade Federal do Estado de São Paulo; 2000.

16. Fukui H. Music and testosterone: a new hypothesis for the origin and function of music. Ann. NY acad. sci. [Internet]. 2001 jun;930:448-51. [acesso em 10 mar 2011]. Disponível: http://onlinelibrary.wiley.com/ doi/10.1111/j.1749-6632.2001.tb05767.x/full

17. Almeida AP. Canto Gregoriano como redutor de ansiedade das mães de crianças hospitalizadas: estratégia para a enfermagem [dissertação]. São Paulo (SP): Universidade de São Paulo; 2010. 